\title{
Tensiomyographical responsiveness to peripheral fatigue in quadriceps femoris (\#44194)
}

First revision

\section{Guidance from your Editor}

Please submit by $\mathbf{7}$ Feb $\mathbf{2 0 2 0}$ for the benefit of the authors .

\section{Structure and Criteria}

Please read the 'Structure and Criteria' page for general guidance.

\section{Custom checks}

Make sure you include the custom checks shown below, in your review.

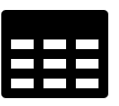

\section{Raw data check}

Review the raw data.

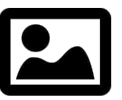

\section{Image check}

Check that figures and images have not been inappropriately manipulated.

Privacy reminder: If uploading an annotated PDF, remove identifiable information to remain anonymous.

\section{Files}

Download and review all files from the materials page.
1 Tracked changes manuscript(s)

1 Rebuttal letter(s)

4 Figure file(s)

3 Table file(s)

1 Raw data file(s)

1 Other file(s)

\section{(? Custom checks}

\section{Human participant/human tissue checks}

Have you checked the authors ethical approval statement?

Does the study meet our article requirements?

Has identifiable info been removed from all files?

Were the experiments necessary and ethical? 


\section{Structure your review}

The review form is divided into 5 sections. Please consider these when composing your review:

\section{BASIC REPORTING}

2. EXPERIMENTAL DESIGN

3. VALIDITY OF THE FINDINGS

4. General comments

5. Confidential notes to the editor

You can also annotate this PDF and upload it as part of your review

When ready submit online.

\section{Editorial Criteria}

Use these criteria points to structure your review. The full detailed editorial criteria is on your guidance page.

\section{BASIC REPORTING}

Clear, unambiguous, professional English language used throughout.

Intro \& background to show context. Literature well referenced $\&$ relevant.

Structure conforms to Peer] standards, discipline norm, or improved for clarity.

Figures are relevant, high quality, well labelled \& described.

Raw data supplied (see Peer] policy).

\section{EXPERIMENTAL DESIGN}

Original primary research within Scope of the journal.

Research question well defined, relevant \& meaningful. It is stated how the research fills an identified knowledge gap.

Rigorous investigation performed to a high technical \& ethical standard.

Methods described with sufficient detail \& information to replicate.

\section{VALIDITY OF THE FINDINGS}

Impact and novelty not assessed.

Negative/inconclusive results accepted. Meaningful replication encouraged where rationale $\&$ benefit to literature is clearly stated.

All underlying data have been provided; they are robust, statistically sound, $\&$ controlled.
Speculation is welcome, but should be identified as such.

Conclusions are well stated, linked to original research question $\&$ limited to supporting results. 


\section{Standout \\ reviewing tips}

The best reviewers use these techniques

Tip

\section{Support criticisms with evidence from the text or from other sources}

\section{Give specific suggestions on how to improve the manuscript}

\section{Comment on language and grammar issues}

\author{
Please provide constructive \\ criticism, and avoid personal \\ opinions
}

\section{Example}

Smith et al (J of Methodology, 2005, V3, pp 123) have shown that the analysis you use in Lines 241-250 is not the most appropriate for this situation. Please explain why you used this method.

Your introduction needs more detail. I suggest that you improve the description at lines 57- 86 to provide more justification for your study (specifically, you should expand upon the knowledge gap being filled).

The English language should be improved to ensure that an international audience can clearly understand your text. Some examples where the language could be improved include lines 23, 77, 121, 128 - the current phrasing makes comprehension difficult.

1. Your most important issue

2. The next most important item

3....

4. The least important points

I thank you for providing the raw data, however your supplemental files need more descriptive metadata identifiers to be useful to future readers. Although your results are compelling, the data analysis should be improved in the following ways: $A A, B B, C C$

I commend the authors for their extensive data set, compiled over many years of detailed fieldwork. In addition, the manuscript is clearly written in professional, unambiguous language. If there is a weakness, it is in the statistical analysis (as I have noted above) which should be improved upon before Acceptance.
Comment on strengths (as well as weaknesses) of the manuscript 


\title{
Tensiomyographical responsiveness to peripheral fatigue in quadriceps femoris
}

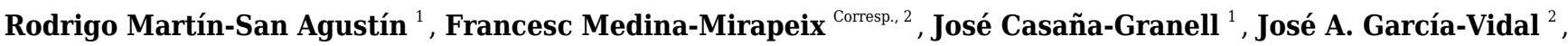 \\ Carmen Lillo-Navarro ${ }^{3}$, Josep C. Benítez-Martínez ${ }^{1}$ \\ ${ }^{1}$ Department of Physiotherapy, University of Valencia, Valencia, España \\ 2 Department of Physiotherapy, University of Murcia, Murcia, Spain \\ 3 Department of Pathology and Surgery, University Miguel Hernández, San Joan, Spain \\ Corresponding Author: Francesc Medina-Mirapeix \\ Email address: mirapeix@um.es
}

Background: Fatigue influences athletic performance and can also increase the risk of injury in sports and most of the methods to evaluate it require an additional voluntary effort. Tensiomyography (TMG), which uses electrical stimulation and a displacement sensor to evaluate muscle contraction properties of one or more muscle bellies, has emerged as a technique that can assess the presence of peripheral and central fatigue without requiring additional voluntary efforts. However, the evaluation of the TMG's ability to detect fatigue is limited, both at the level of muscle bellies and statistical methods. Thus, the aim of the present study was twofold: (i) to examine and compare the tensiomyographical responsiveness to quadriceps femoris (QF) fatigue by multiple statistical methods and (ii) to analyze sex differences in the variation produced by fatigue in TMG parameters. Methods: Thirty-nine recreational athletes participated (19 males/20 females; aged $22 \pm 2$ years). TMG parameters of QF bellies and maximal voluntary isometric contraction (MVIC) were measured before and after a fatigue protocol. TMG parameters used were maximum radial deformation $(\mathrm{Dm})$, contraction time between $10-90 \%$ of the Dm (Tc), contraction velocity between $10-90 \%(\mathrm{Vc})$ and of the first $10 \%$ (V10) of the Dm. Internal responsiveness of TMG to fatigue was analyzed by paired t-test and standardized response mean (SRM). External responsiveness was examined by correlations, regression models, and receiver operating characteristic (ROC) curves.

Results: All TMG parameters, except for Tc of rectus femoris and vastus medialis, showed large internal responsiveness. In adjusted regression models by sex, only Dm and V10 of rectus femoris were statistically associated $(p<0.05)$ with b coefficients of 0.40 and 0.43 , respectively. $r 2$ explained the $22 \%$ of the total variance. In addition, these parameters could discriminate between QF with and without fatigue. Conclusion: Since the QF is the main strength contributor during multiple physical activities, clinicians and trainers will be 
able to discriminate the presence of fatigue and the magnitude of changes in the QF strength by TMG evaluation. 


\section{Tensiomyographical responsiveness to peripheral 2 fatigue in quadriceps femoris}

3

4

5

6

7

8 9

10

11

12

13

14

15

16

17

18

19

20

21

22

23

24

25

26

27

28

29

30

31

32

33

34

35

36

37

38

Rodrigo Martín-San Agustín ${ }^{1}$, Francesc Medina-Mirapeix ${ }^{2}$, José Casaña-Granel1 ${ }^{1}$, José A. García-Vidal $^{2}$, Carmen Lillo-Navarro ${ }^{3}$, Josep C. Benítez-Martínez ${ }^{1}$

${ }^{1}$ Department of Physiotherapy, University of Valencia, Valencia, Spain

2 Department of Physiotherapy, University of Murcia, Murcia, Spain

${ }^{3}$ Department of Pathology and Surgery. University Miguel Hernández, Sant Joan, Spain

Corresponding Author:

Francesc Medina-Mirapeix

Department of Physiotherapy - Faculty of Medicine, University of Murcia, Campus de Espinardo, Murcia 30100, Spain.

Email address: mirapeix@um.es

\section{Abstract}

Background: Fatigue influences athletic performance and can also increase the risk of injury in sports and most of the methods to evaluate it require an additional voluntary effort.

Tensiomyography (TMG), which uses electrical stimulation and a displacement sensor to evaluate muscle contraction properties of one or more muscle bellies, has emerged as a technique that can assess the presence of peripheral and central fatigue without requiring additional voluntary efforts. However, the evaluation of the TMG's ability to detect fatigue is limited, both at the level of muscle bellies and statistical methods. Thus, the aim of the present study was twofold: (i) to examine and compare the tensiomyographical responsiveness to quadriceps femoris (QF) fatigue by multiple statistical methods and (ii) to analyze sex differences in the variation produced by fatigue in TMG parameters. Methods: Thirty-nine recreational athletes participated (19 males/20 females; aged $22 \pm 2$ years). TMG parameters of QF bellies and maximal voluntary isometric contraction (MVIC) were measured before and after a fatigue protocol. TMG parameters used were maximum radial deformation $(\mathrm{Dm})$, contraction time between $10-90 \%$ of the $\mathrm{Dm}(\mathrm{Tc})$, contraction velocity between $10-90 \%(\mathrm{Vc})$ and of the first $10 \%$ (V10) of the Dm. Internal responsiveness of TMG to fatigue was analyzed by paired t-test and standardized response mean (SRM). External responsiveness was examined by correlations, regression models, and receiver operating characteristic (ROC) curves.

Results: All TMG parameters, except for Tc of rectus femoris and vastus medialis, showed large internal responsiveness. In adjusted regression models by sex, only Dm and V10 of rectus femoris were statistically associated $(p<0.05)$ with $b$ coefficients of 0.40 and 0.43 , respectively. $\mathrm{r} 2$ explained the $22 \%$ of the total variance. In addition, these parameters could discriminate between QF with and without fatigue. Conclusion: Since the QF is the main strength contributor 
39

40

41

\section{2}

43

44

45

46

47

48

49

50

51

52

53

54

55

56

57

58

59

60

61

62

63

64

65

66

67

68

69

70

71

72

73

74

75

76

77

78

during multiple physical activities, clinicians and trainers will be able to discriminate the presence of fatigue and the magnitude of changes in the QF strength by TMG evaluation.

\section{Introduction}

Fatigue is defined as a decline in muscular performance which produces a reduction in strength and power generation (Ditroilo et al., 2011). It can be further explained by factors related to the central nervous system as changes at the spinal level (Gandevia, 2001) or by peripheral factors associated to the muscle, such as failure of transmission at the neuromuscular junction (Allen, Lamb \& Westerblad, 2008). Its manifestation can vary in subjects with different training backgrounds (Garrandes et al., 2007), type of muscle contraction performed (Kay et al., 2000), or even between sex (Albert et al., 2006; Martin \& Rattey, 2007; Ansdell et al., 2017).

Since fatigue influences athletic performance (Thorlund et al., 2008; Ditroilo et al., 2011) and can also increase the risk of injury in sports (Zebis et al., 2011; Liederbach et al., 2014), its study has been of interest. Multiple methods have been used to induce fatigue, both central fatigue in several muscle groups or peripheral fatigue in a specific muscle (García-Manso et al., 2011; Hunter et al., 2012; Macgregor et al., 2016; Wiewelhove et al., 2017, 2018). Thus, fatigue has been evaluated after short term (Macgregor et al., 2016; Abelairas-Gómez et al., 2018) and long duration efforts, such as several days of intense training sessions (Wiewelhove et al., 2017), and also after isolated long sessions (2-12h approximately) (Lepers et al., 2002; García-Manso et al., 2011; Wiewelhove et al., 2018).

The most used fatigue evaluation methods have been based on changes in maximal voluntary isometric contractions (MVICs) (Lepers et al., 2002; Zebis et al., 2011), muscle activation (Garrandes et al., 2007; Thorlund et al., 2008), kinematics and kinetics measurements (Liederbach et al., 2014; Tam et al., 2017), biochemical markers (Gorostiaga et al., 2012), or muscular contractile properties (García-Manso et al., 2011; de Paula Simola et al., 2016). In a situation of fatigue, most of these methods would require an additional voluntary effort. Their application therefore would not be practical or safe facing the possible presence of central inhibition (Graven-Nielsen et al., 2002), or the possibility of increase any extant muscular damage (Macgregor et al., 2016).

Tensiomyography (TMG), which uses electrical stimulation and a displacement sensor to evaluate muscle contraction properties of one or more muscle bellies (Valencic \& Knez, 1997), has emerged as a technique that can assess the presence of peripheral and central fatigue without requiring additional voluntary efforts (García-Manso et al., 2011; de Paula Simola et al., 2016). Peripheral fatigue has been evaluated by TMG for specific muscle group from both lower and upper limbs (Carrasco et al., 2011; Hunter et al., 2012; García-Manso et al., 2012; Macgregor et al., 2016). In contrast, central fatigue has been evaluated only in the lower limb, being quadriceps femoris (QF) the most studied muscle group (García-Manso et al., 2011; de Paula Simola et al., 2015, 2016; Giovanelli et al., 2016; Raeder et al., 2016; Wiewelhove et al., 2017). Responsiveness is defined as the ability of a tool to detect important clinical changes over time (Guyatt et al., 1989). Since this characteristic is essential to assess fatigue by TMG, it has been 
79

80

81

82

83

84

85

86

87

88

89

90

91

92

93

94

95

96

97

98

99

100

101

102

103

104

105

106

107

108

109

110

111

112

113

114

115

116

117

analyzed by multiple studies (García-Manso et al., 2011; Hunter et al., 2012; de Paula Simola et al., 2015, 2016; Giovanelli et al., 2016; Macgregor et al., 2016; Raeder et al., 2016; Wiewelhove et al., 2017; Abelairas-Gómez et al., 2018). Most of these studies evaluated one muscle belly and they used one or two statistical methods of either internal responsiveness (e.g. paired t-test and effect size) or external responsiveness (correlation with reference measure or regression models) Internal responsiveness is the ability of a measure to change over a set period and external responsiveness reflects the extent to which changes in a measure over a specified time frame related to corresponding changes in an external reference measure of health status (Husted et al., 2000). Overall, TMG of those evaluated muscle bellies has shown to be internally and externally responsive in assessing central fatigue (García-Manso et al., 2011; de Paula Simola et al., 2015, 2016; Giovanelli et al., 2016; Raeder et al., 2016; Wiewelhove et al., 2017), and internally responsive to peripheral fatigue (Hunter et al., 2012; García-Manso et al., 2012; Macgregor et al., 2016; Abelairas-Gómez et al., 2018). However, to the best of our knowledge, the external responsiveness of TMG has not been yet assessed for peripheral fatigue, and therefore comparisons between internal and external responsiveness has not been established. Furthermore, to our knowledge, TMG responsiveness has not been simultaneously evaluated in multiple bellies, neither analyzed by by multiple statistical indicators of responsiveness. At the same time, understanding the mechanisms behind the changes in TMG parameters caused by fatigue in both sexes, is also an area of research that needs further development.

Therefore, the primary objective of our study was to examine and compare the responsiveness of TMG parameters to QF peripheral fatigue of three muscle bellies [rectus femoris (RF), vastus lateralis (VL), and vastus medialis (VM)] by multiple statistical methods. A secondary objective was to examine whether there are differences between sex in the variation produced by fatigue in TMG parameters. Our hypotheses were: QF bellies have different responsiveness to peripheral fatigue; and the changes of TMG parameters are similar between males and females.

\section{Materials \& Methods} Study design

A single group pretest-posttest design was used, which involved repeated TMG and MVIC measures of the dominant lower limb QF before and after a fatigue protocol within the same session. Participants were physiotherapy students recruited by email using the University of Valencia Intranet. This study was conducted from April to July 2018. All measurements were carried out between 10 a.m. and 2 p.m in the clinical research laboratory of the Department of Physiotherapy (University of Valencia) at an ambient temperature $21-22^{\circ} \mathrm{C}$. An experienced examiner in the measurement techniques evaluated the participants. He was a physiotherapist who had used TMG and hand dynamometers both in research and in clinical practice for several years. Before participation, participants were informed of the study procedures and their possible associated risks. All of them provided written informed consent. This study was completed 
118 following the principles outlined in the Declaration of Helsinki and it was approved by the Ethics

119 Committee of the University of Valencia (Spain) (H1523633864087).

120

121

\section{Participants}

122

Thirty-nine recreational athletes were evaluated. All participants performed exercise 3 times per

123 week and practiced activities such as running, swimming, cycling, or central strength training. The specific inclusion criteria were: (a) aged between 18 and 30 years, (b) not surgically operated on the lower limb, (c) without pain in the lower limb in the 2 months before data collection, and (d) performing physical exercise a minimum of 3 days per week. The exclusion criteria were: (a) practicing a specific sport as an amateur or professional, (b) contraindication to the use of electrodes due to injury or allergy to the adhesive, and (c) nontolerance to electrical stimulation.

130

\section{Procedures}

Before starting the session, height was measured using a 1-millimeter sensitivity flexible tape measure, while body mass and body mass index (BMI) were assessed using a standardized body composition analyzer (Tanita BC 418 MA, Tanita Corp, Tokyo, Japan). Next, TMG parameters were measured and then, participants performed a warm-up, which consisted of 10 minutes cycling at comfortable speed ( 80 revolutions per minute) with low resistance and the performance of three submaximal isometric contractions of isometric knee extension (Martins et al., 2017). Following this, the MVIC test was performed. After the fatigue protocol, the order of the tests was reversed, and the strength test was performed first to reduce the time between MVIC and TMG tests in acute fatigue. A schematic representation of the experimental procedures is reported in the Figure 1.

142

\section{Tensiomyography measurements}

First, participants were placed supine and resting on the stretcher. The knee was placed at $120^{\circ}$ of flexion (considering full extension at $180^{\circ}$ ), fixing such position with a triangular foam cushion (García-García et al., 2016; Martín-San Agustín et al., 2018). The area where the TMG sensor and electrodes were placed was shaved and cleaned with gauze and alcohol. The position of the sensor for each QF belly was determined using the anatomical criteria described in the literature (Dahmane et al., 2005; Tous-Fajardo et al., 2010; Rey, Lago-Peñas \& Lago-Ballesteros, 2012). This position was marked with a permanent marker so that it would remain throughout the evaluation. The sensor was finally placed on this point perpendicularly to the thigh and the electrodes were placed at $5 \mathrm{~cm}$ distance from it, forming an imaginary straight line along the 
159

160

161

162

163

164

165

166

167

168

169

170

171

172

173

174

175

176

177

178

179

180

181

182

183

184

185

186

187

188

189

190

191

192

193

194

195

196

197

198

The contractile properties of each belly were evaluated during an maximal elicited contractions with the TMG electro stimulator (TMG-100 System). Starting from $20 \mathrm{~mA}$ with $1 \mathrm{~ms}$ pulses, each stimulation was increased by $10 \mathrm{~mA}$ until achieving the maximum radial deformation (Dm) of the muscular belly. A time of 10s was left between stimuli to minimize fatigue or potentiation effects (Krizaj, Simunic \& Zagar, 2008). Before data acquisition, a pilot test was done to verify the functioning of the TMG. For each belly, spatial and temporal parameters were measured: $\mathrm{Dm}$, contraction time between 10 and $90 \%$ of the Dm (Tc), contraction velocity between 10 and $90 \%$ of the Dm (Vc), and contraction velocity of the first $10 \%$ of the Dm (V10). TMG has proven to be a method with a high relative [ICC for Dm (0.91-0.99), Tc (0.70-0.98), and VC > 0.95] and absolute (low coefficient of variations for Dm, Tc, and VC) reliability (MartínRodríguez et al., 2017; Lohr et al., 2018).

\section{Maximal voluntary isometric contraction test}

MVIC of the QF was measured by a MicroFET2 handheld dynamometer (Hoggan Health Technologies Inc., Salt Lake City, UT). Participants were seated in an isokinetic dynamometer (Prima Plus, Easytech, Italy) with their torso and hips tied so they were stable, and with a $90^{\circ}$ hip flexion. MVIC was evaluated in $90^{\circ}$ knee flexion, considering $0^{\circ}$ the complete extension (Figure 3). MicroFET2 was fixed with a rigid belt perpendicular to the ankle $5 \mathrm{~cm}$ above the malleoli, with a pad between the tibia and the dynamometer to minimize the discomfort caused by the contact (Hansen et al., 2015).

After the warm-up, participants completed three MVIC for $5 \mathrm{~s}$, with a 60 -second rest after each repetition. Through verbal stimuli, participants were instructed to exert and maintain the maximum effort during the session. MicroFET2 has proven to be a valid method to measure the MVIC of the QF with an excellent inter-examiner reliability (ICC: $0.93,95 \%$ CI 0.83; 0.97) and a minimal detectable change (MDC) of $14.1 \mathrm{~N}^{*} \mathrm{~m}$ (95\% CI, 9.23; 22.01) (Hansen et al., 2015).

\section{[Please insert Figure 3 about here]}

\section{Fatigue protocol test}

After performing the baseline measurements, participants were requested to implement a protocol based on a 60s fatiguing isometric contraction at 70\% MVC (Melchiorri \& Rainoldi, 2011). The experimental setup was the same as the one adopted during the MVIC test. The handheld dynamometer, previously set at 70\% MVIC, was used to display the feedback (Melchiorri \& Rainoldi, 2011). It was considered that the fatigue was achieved when the reduction of the MVIC was higher than the upper limit of the MDC reported in a previous study $\left(22.01 \mathrm{~N}^{*} \mathrm{~m}\right)$ (Hansen et al., 2015).

\section{Statistical analysis}


199 Baseline data were summarized as means and standard deviations (SD) for continuous variables

200

201

202

203

204

205

206

207

208

209

210

211

212

213

214

215

216

217

218

219

220

221

222

223

224

225

226

227

228

229

230

231

232

233

234

235

236

237

238

and as absolute and relative frequencies for categorical variables. Variables were checked for normality with the Kolmogorov-Smirnov test and homogeneity of variances with Levene's test. A summary was also provided for participants with and without fatigued QF.

Paired t-tests were used to compare changes in the TMG parameters and MVIC within each sex group. These changes were also compared between sex groups by using non-paired t-tests. Internal responsiveness was determined by the paired t-test and supplemented with an effect size statistic, as recommended by Husted et al. (2000) [30]. To reduce the probability of getting false positives, we increased the acceptance level from 0.05 to 0.01 for paired t-test because multiple comparisons were made on the same data set. Of the current effect size statistics we used the standardized response mean (SRM), which provides an estimate of the magnitude of change that is not influenced by sample size (Navarro-Pujalte et al., 2018). It was calculated as

(MeanFollowup_MeanBaseline)/Standard deviationFollowup-Baseline and the $95 \%$ confidence intervals were calculated using the bootstrapping estimation method. Values of $0.20,0.50$, and 0.80 or higher have been proposed in the literature (Husted et al., 2000) to represent small, moderate, and large responsiveness, respectively. Besides, we calculated the percentage of participants that exceeded MDC. This statistic examines the extent to which change score exceeds the amount of variability accounted by measurement error (Pardasaney et al., 2012), which is calculated as $S E M \times 1.96 x \sqrt{2}$, where SEM is the standard error of measurement. External responsiveness was determined by correlations, regression models, and receiver operating characteristic (ROC) curves (Husted et al., 2000). The external criterion for assessing the external responsiveness of the TMG tool was the magnitude of change in the MVIC. We assumed that: (i) changes in the external criterion (MVIC) in participants with fatigue would be associated with changes in the TMG parameters; (ii) participants without fatigue would have the smallest change in the TMG parameters (and therefore change in these TMG parameters can be useful to classify participants' QF as fatigued or not fatigued). To test the first hypothesis, correlations and simple and multiple linear regression models were used. In the regression models the explanatory variable was the change of each TMG parameter while the response variable was the change in MVIC between before and after protocols. Each model was controlled by sex, and comparisons were carried out between the presence or absence of this control. Goodness-of-fit of the model was assessed by $\mathrm{r} 2$. To test the second hypothesis, we calculated the area under the ROC curve (AUC), which represents the probability that the measure of correctly classifying participants has (Husted et al., 2000). An AUC $>0.70$ was used as a generic benchmark to consider acceptable its discriminant ability (Menaspà, Sassi \& Impellizzeri, 2010). For sample size calculation, we selected the multiple regression as the main statistic of responsiveness because it allowed us to examine change relationships controlling by a covariate relevant in our study (sex). Regarding this statistic, we used the usual rule of thumb that 15 participants per predictor are needed for a reliable equation in multiple regression models (Tabachnick \& Fidell, 2007). We recruited a minimum of 30 participants assuming a maximum of 2 explanatory variables (TMG parameter and sex). Statistical significance was set at $p<0.05$.

Peer) reviewing PDF | (2019:12:44194:1:1:NEW 23 Jan 2020) 
239

240

241

242

243

244

245

246

247

248

249

250

251

252

253

254

255

256

257

258

259

260

261

262

263

264

265

266

267

268

269

270

271

272

273

274

275

276

277

All analyses were performed using the Statistical Package for the Social Sciences software program (SPSS version 24.0; IBM SPSS, Chicago, IL, USA).

\section{Results}

\section{Participants' characteristics}

Baseline characteristics of participants are listed in Table 1. A total of 35 (89.7\%) participants achieved QF fatigue after the application of the fatigue protocol. They were 19 of 20 females (95\%) and 16 of 19 males (84.2\%). Participants with and without fatigue showed no significant differences $(p>0.05)$ in any of their baseline characteristics.

\section{[Please insert Table 1 about here]}

\section{Changes associated with the fatigue protocol}

Participants with peripheral fatigue $(n=35)$ had a significant decrease $(31.5 \%)$ on their MVIC after the fatigue protocol (from $203.3 \mathrm{~N}^{*} \mathrm{~m}$ to $138.9 \mathrm{~N}^{*} \mathrm{~m}$ ). Table 2 shows that both sex groups had a similar pattern of change: males reduced $30.8 \%$ and females $32.1 \%$. Table 2 also shows patterns of change by sex groups for TMG parameters of the RF, VL, and VM. All these parameters, except for the Tc of the RF and VM, had significant differences within but not between sex groups.

Figure 4 shows changes in TMG parameters for all participants with peripheral fatigue. All parameters, except for Tc, showed a significant difference $(p<0.001)$ for the three bellies of the QF. Dm's decrease ranged from $18.22 \%$ to $21.65 \%$; Vc decreased from 15.62 to $22.20 \%$, and V10 decreased from $14.80 \%$ to $23.77 \%$.

\section{[Please insert Table 2 about here]}

\section{[Please insert Figure 4 about here]}

\section{Internal and external responsiveness}

Internal and external TMG responsiveness to fatigue of QF bellies is shown in Table 3. Internal responsiveness statistics suggest that all TMG parameters, except for Tc of RF and VM, showed large internal responsiveness (SRM $>0.8$ ) among participants with QF fatigue. Dm and V10 in RF were the parameters in which most of the participants exceeded the MCD $(91.3 \%$ and $97.1 \%$, respectively). Only Dm, Vc, and V10 of the RF showed to be linearly associated with changes in the MVIC. After controlling by sex, adjusted models typically provided b coefficients and $\mathrm{r} 2$ with small variations regarding their respective unadjusted model (range 0.01 to 0.05 ). Consequently, Dm and V10 of RF were still statistically associated with b coefficients of 0.40 and 0.43 , respectively. Moreover, the models of these parameters explained the $22 \%$ of the total variance. 
278 The AUC analysis suggests that changes of several TM G parameters (Dm in RF and VL, Tc in 279 VL, and V10 in RF and VM) were $>0.70$ and could discriminate between QF with and without 280 fatigue. Also, the overlapping among their $95 \%$ CI suggests that none of these TMG parameters 281 is superior to the others to discriminate fatigue.

282

283

284

285

286

287

288

289

290

291

292

293

294

295

296

297

298

299

300

301

302

303

304

305

306

307

308

309

310

311

312

313

314

315

316

317

\section{[Please insert Table 3 about here]}

\section{Discussion}

To our knowledge, this is the first study to evaluate the internal and external TMG

responsiveness across a variety of QF muscle bellies to changes induced by peripheral fatigue. We found that TMG parameters Dm and V10 of the RF showed both internal and external responsiveness.

In our study, multiple statistical methods to evaluate the internal responsiveness (paired t-test and SRM) and external responsiveness (correlations, regression models and ROC) of the TMG were used, which is line with the recommendations of Husted et al. (2000). In previous studies, most of these statistics have been used to evaluate only the TMG ability of change to fatigue (GarcíaManso et al., 2011; de Paula Simola et al., 2015). Thus, to the best of our knowledge, this is the first study to use several statistical methods to assess internal and external responsiveness. Furthermore, since most of the previous studies assessing fatigue by TMG have only evaluated isolated muscle bellies (García-Manso et al., 2011; Hunter et al., 2012; de Paula Simola et al., 2015, 2016; Giovanelli et al., 2016; Macgregor et al., 2016; Raeder et al., 2016; Wiewelhove et al., 2017) ), our study presents novel findings in the evaluation of TMG across multiple muscle bellies.".

Regarding the internal responsiveness, large and negative SRM of the TMG parameters were found in most of the muscle bellies. Overall, our results are consistent with previous studies that induced peripheral and central $\mathrm{QF}$ fatigue (i.e. selective $\mathrm{QF}$ fatigue or caused in the entire lower limb musculature). Therefore, the reduction of RF TMG parameters is consistent with previous studies using peripheral (Carrasco et al., 2011) or central fatigue (de Paula Simola et al., 2015), finding them reductions in $\mathrm{Dm}, \mathrm{VC}$, or V10 after fatigue due to cycling or strengthening. On the other hand, the changes in VL and VM are also consistent with studies using central fatigue caused by strengthening programs (de Paula Simola et al., 2016; Raeder et al., 2016). In addition, Dm results showed consistence with other studies that induced peripheral fatigue in muscles such as the biceps brachii (Hunter et al., 2012; García-Manso et al., 2012) or the gastrocnemius medialis (Macgregor et al., 2016). These findings could be explained by changes in the $\mathrm{pH}$ (Hunter et al., 2009) and in different cellular molecules (e.g. Na+ or K+) (Brody et al., 1991), which cause damage in the sarcolemma and the reduction of the electrical stimulus, with a possible decrease in muscle displacement.

This study showed that Dm and V10 of RF had an acceptable external responsiveness in relation to our external criterion, namely changes in the strength evidenced by MVIC. As reflected by the 
318 regression coefficients, there was a moderate relationship between the amount of change in TMG 319 parameters and strength scores. This relationship is consistent with a previous study using central 320 fatigue (de Paula Simola et al., 2015). Furthermore, Dm and V10 were relevant according to sex, 321 which can be explained by the fact that our sample showed similar change magnitudes in both 322 TMG parameters and strength scores.

323 The fatigue protocol used in this study was highly effective (most of the QF showed fatigue).

324 Males and females had similar strength change scores (Table 2). Previous studies reported 325 different strength change scores between sexes when intensities between 25-50\% of MVIC were

326

327

328

329

330

331

332

333

334

335

336

337

338

339

340

341

342

343

344

345

346

347

348

349

350

351

352

353

354

355

356

357 used (Clark et al., 2005; Ansdell et al., 2017). In our study, an intensity of 70\% of MVIC was used, suggesting that as the contraction intensity increase, the sex differences in muscle fatigue decrease, (Hunt , 2014). Therefore, future investigations should examine whether sex differences in strength changes are detected by sex differences in the TMG changes.

Our present study also showed that TMG has discriminative ability to classify the participants' QF as having fatigue or not after the application of the protocol. Dm and V10 of the RF also were two of the four parameters with this discriminative ability. This finding is partially consistent with previous studies (Wiewelhove et al., 2017), who examined AUC of RF after central fatigue in elite young athletes. Nevertheless, while AUC values of V10 shown in this study was similar to their results, AUC values of Dm was higher than previously published (Wiewelhove et al., 2017). Differences may be explained by the different type of fatigue (central fatigue caused by several training sessions of high-intensity interval training vs peripheral fatigue by an MVIC test) or by the athletes' training background (junior tennis players vs recreational athletes). Other parameters with that discriminative ability were Dm and Tc of VL, and V10 of VM. Since this ability was not previously analyzed in these muscle bellies (VL and VM), results of the actual study supplements earlier findingswhich have only evaluated AUC for external responsiveness of the TMG in RF (Wiewelhove et al., 2017) and it provides evidence to expand the application of the TMG to discriminate fatigue.

Actual study has several limitations. First, we used a fatigue protocol based on MVIC, which induces peripheral fatigue. Therefore, our findings would be limited to be extrapolated to others fatigue situations (e.g. concentric contractions). Second, our study was conducted with recreational athletes (i.e. anyone participating in an aerobic or athletic activity at least three times per week) (Heinert et al., 2008). Since the contractile properties of the muscle are conditioned by the type of exercise performed (Loturco et al., 2015), future research should compare our results with findings from athletes of different sports.

Our study found that most of the TMG parameters showed an acceptable internal responsiveness of QF peripheral fatigue evidenced by a reduction of the MVIC. In contrast, only Dm and V10 of RF showed external responsiveness. Therefore, our study illustrates that the use of only internal or external responsiveness may lead to incomplete conclusions (Husted et al., 2000). In this way, professionals should use both, as recommended by Husted (Husted et al., 2000).

This study showed that Dm and V10 of RF measured by TMG were both internally and externally responsive to changes between before and after a peripheral fatigue protocol. Since the 
358 QF is the main strength contributor during cycling (Raasch et al., 1997) or running

359 (Montgomery, Pink \& Perry, 1994), the fatigue evaluation after an effort is essential to manage

360 recovery of the athlete and the intensity of subsequent training sessions. Thus, clinicians and

361 trainers should be able to direct the fatigue evaluations without making new efforts with TMG,

362 taking into consideration Dm and V10 parameters in RF to discriminate the presence of

363 peripheral fatigue and the magnitude of the strength changes and, in this way, be able to regulate

364 training loads (e.g. in the presence of peripheral fatigue, decrease intensity or activities that

365 involve the QF).

366

\section{Conclusions}

368 According to the results, it can be concluded about positive responsiveness of the TMG in 369 peripheral fatigue of the QF, demonstrating that the Dm and V10 parameters of the RF present

370 acceptable responsiveness to fatigue. Therefore, by using the TMG, it is possible to determine

371 whether the QF shows peripheral fatigue or not, and to relate changes in the parameters with the

372 reduction of strength. Thus, clinicians and trainers should be able to direct the fatigue evaluations

373 without making new efforts with TMG, facilitating the regulation of training loads. Finally,

374 future studies should examine the responsiveness of TMG to other types of fatigue and in other

375 sports.

376

377

378

379

380

381

382

\section{References}

Abelairas-Gómez C, Rey E, González-Salvado V, Mecías-Calvo M, Rodríguez-Ruiz E,

Rodríguez-Núñez A. 2018. Acute muscle fatigue and CPR quality assisted by visual feedback devices: A randomized-crossover simulation trial. PLOS ONE 13. DOI:

Albert W, Wrigley A, McLean R, Sleivert G. 2006. Sex differences in the rate of fatigue development and recovery. Dynamic Medicine 5:2. DOI: 10.1186/1476-5918-5-2.

Allen DG, Lamb GD, Westerblad H. 2008. Skeletal muscle fatigue: cellular mechanisms. Physiological Reviews 88:287-332. DOI: 10.1152/physrev.00015.2007. 

DOI: $10.1016 /$ j.jelekin.2017.09.003.

395

Brody LR, Pollock MT, Roy SH, De Luca CJ, Celli B. 1991. pH-induced effects on median frequency and conduction velocity of the myoelectric signal. Journal of Applied Physiology (Bethesda, Md.: 1985) 71:1878-1885. DOI: 10.1152/jappl.1991.71.5.1878.

Carrasco L, Sañudo B, de Hoyo M, Pradas F, Da Silva ME. 2011. Effectiveness of lowfrequency vibration recovery method on blood lactate removal, muscle contractile properties and on time to exhaustion during cycling at $\mathrm{VO}_{2}$ max power output. European Journal of Applied Physiology 111:2271-2279. DOI: 10.1007/s00421-011-1848-9.

Clark BC, Collier SR, Manini TM, Ploutz-Snyder LL. 2005. Sex differences in muscle fatigability and activation patterns of the human quadriceps femoris. European Journal of Applied Physiology 94:196-206. DOI: 10.1007/s00421-004-1293-0.

Dahmane R, Djordjevic S, Simunic B, Valencic V. 2005. Spatial fiber type distribution in normal human muscle Histochemical and tensiomyographical evaluation. Journal of Biomechanics 38:2451-2459. DOI: 10.1016/j.jbiomech.2004.10.020.

Ditroilo M, Watsford M, Fernández-Peña E, D'Amen G, Lucertini F, De Vito G. 2011. Effects of fatigue on muscle stiffness and intermittent sprinting during cycling. Medicine and Science in Sports and Exercise 43:837-845. DOI: 10.1249/MSS.0b013e3182012261.

Gandevia SC. 2001. Spinal and supraspinal factors in human muscle fatigue. Physiological Reviews 81:1725-1789. DOI: 10.1152/physrev.2001.81.4.1725.

García-García O, Serrano-Gómez V, Hernández-Mendo A, Tapia-Flores A. 2016. Assessment of the in-season changes in mechanical and neuromuscular characteristics in professional soccer players. The Journal of Sports Medicine and Physical Fitness 56:714-723.

García-Manso JM, Rodríguez-Matoso D, Sarmiento S, de Saa Y, Vaamonde D, Rodríguez-Ruiz D, Da Silva-Grigoletto ME. 2012. Effect of high-load and high-volume resistance 
419

420

421

422

423

424

425

426

427

428

429

430

431

432

433

434

435

436

437

438

439

440

441

442

443 exercise on the tensiomyographic twitch response of biceps brachii. Journal of Electromyography and Kinesiology: Official Journal of the International Society of Electrophysiological Kinesiology 22:612-619. DOI: 10.1016/j.jelekin.2012.01.005.

García-Manso JM, Rodríguez-Ruiz D, Rodríguez-Matoso D, de Saa Y, Sarmiento S, Quiroga M. 2011. Assessment of muscle fatigue after an ultra-endurance triathlon using tensiomyography (TMG). Journal of Sports Sciences 29:619-625. DOI: 10.1080/02640414.2010.548822.

Garrandes F, Colson SS, Pensini M, Seynnes O, Legros P. 2007. Neuromuscular fatigue profile in endurance-trained and power-trained athletes. Medicine and Science in Sports and Exercise 39:149-158. DOI: 10.1249/01.mss.0000240322.00782.c9.

Giovanelli N, Taboga P, Rejc E, Simunic B, Antonutto G, Lazzer S. 2016. Effects of an Uphill Marathon on Running Mechanics and Lower-Limb Muscle Fatigue. International Journal of Sports Physiology and Performance 11:522-529. DOI: 10.1123/ijspp.2014-0602.

Gorostiaga EM, Navarro-Amézqueta I, González-Izal M, Malanda A, Granados C, Ibáñez J, Setuain I, Izquierdo M. 2012. Blood lactate and sEMG at different knee angles during fatiguing leg press exercise. European Journal of Applied Physiology 112:1349-1358. DOI: $10.1007 / \mathrm{s} 00421-011-2090-1$.

Graven-Nielsen T, Lund H, Arendt-Nielsen L, Danneskiold-Samsøe B, Bliddal H. 2002. Inhibition of maximal voluntary contraction force by experimental muscle pain: a centrally mediated mechanism. Muscle \& Nerve 26:708-712. DOI: 10.1002/mus.10225.

Guyatt GH, Deyo RA, Charlson M, Levine MN, Mitchell A. 1989. Responsiveness and validity in health status measurement: a clarification. Journal of Clinical Epidemiology 42:403-408.

Hansen EM, McCartney CN, Sweeney RS, Palimenio MR, Grindstaff TL. 2015. Hand-held Dynamometer Positioning Impacts Discomfort During Quadriceps Strength Testing: A Validity and Reliability Study. International Journal of Sports Physical Therapy 10:62-68.

Peer] reviewing PDF | (2019:12:44194:1:1:NEW 23 Jan 2020) 
444 Heinert BL, Kernozek TW, Greany JF, Fater DC. 2008. Hip abductor weakness and lower 445 extremity kinematics during running. Journal of Sport Rehabilitation 17:243-256.

446 Hunter SK. 2014. Sex differences in human fatigability: mechanisms and insight to physiological 447 responses. Acta Physiologica (Oxford, England) 210:768-789. DOI:

$448 \quad$ 10.1111/apha.12234.

449 Hunter AM, De Vito G, Bolger C, Mullany H, Galloway SDR. 2009. The effect of induced 450 alkalosis and submaximal cycling on neuromuscular response during sustained isometric contraction. Journal of Sports Sciences 27:1261-1269. DOI: 10.1080/02640410903165077.

Hunter AM, Galloway SDR, Smith IJ, Tallent J, Ditroilo M, Fairweather MM, Howatson G. 2012. Assessment of eccentric exercise-induced muscle damage of the elbow flexors by tensiomyography. Journal of Electromyography and Kinesiology: Official Journal of the International Society of Electrophysiological Kinesiology 22:334-341. DOI: 10.1016/j.jelekin.2012.01.009.

458

459

460

461

462

463

464

465

466

467

Husted JA, Cook RJ, Farewell VT, Gladman DD. 2000. Methods for assessing responsiveness: a critical review and recommendations. Journal of Clinical Epidemiology 53:459-468.

Kay D, St Clair Gibson A, Mitchell MJ, Lambert MI, Noakes TD. 2000. Different neuromuscular recruitment patterns during eccentric, concentric and isometric contractions. Journal of Electromyography and Kinesiology: Official Journal of the International Society of Electrophysiological Kinesiology 10:425-431.

Krizaj D, Simunic B, Zagar T. 2008. Short-term repeatability of parameters extracted from radial displacement of muscle belly. Journal of Electromyography and Kinesiology: Official Journal of the International Society of Electrophysiological Kinesiology 18:645-651. DOI: 10.1016/j.jelekin.2007.01.008. 
468 Lepers R, Maffiuletti NA, Rochette L, Brugniaux J, Millet GY. 2002. Neuromuscular fatigue

469

470

471

472

473

474

475

476

477

478

479

480

481

482

483

484

485

486

487

488

489

490

491

492 during a long-duration cycling exercise. Journal of Applied Physiology 92:1487-1493. DOI: 10.1152/japplphysiol.00880.2001.

Liederbach M, Kremenic IJ, Orishimo KF, Pappas E, Hagins M. 2014. Comparison of landing biomechanics between male and female dancers and athletes, part 2: Influence of fatigue and implications for anterior cruciate ligament injury. The American Journal of Sports Medicine 42:1089-1095. DOI: 10.1177/0363546514524525.

Lohr C, Braumann K-M, Reer R, Schroeder J, Schmidt T. 2018. Reliability of tensiomyography and myotonometry in detecting mechanical and contractile characteristics of the lumbar erector spinae in healthy volunteers. European Journal of Applied Physiology 118:13491359. DOI: $10.1007 / \mathrm{s} 00421-018-3867-2$.

Loturco I, Gil S, Laurino CF de S, Roschel H, Kobal R, Cal Abad CC, Nakamura FY. 2015. Differences in muscle mechanical properties between elite power and endurance athletes: a comparative study. Journal of Strength and Conditioning Research 29:17231728. DOI: 10.1519/JSC.0000000000000803.

Macgregor LJ, Ditroilo M, Smith IJ, Fairweather MM, Hunter AM. 2016. Reduced Radial Displacement of the Gastrocnemius Medialis Muscle After Electrically Elicited Fatigue. Journal of Sport Rehabilitation 25:241-247. DOI: 10.1123/jsr.2014-0325.

Martin PG, Rattey J. 2007. Central fatigue explains sex differences in muscle fatigue and contralateral cross-over effects of maximal contractions. Pflugers Archiv: European Journal of Physiology 454:957-969. DOI: 10.1007/s00424-007-0243-1.

Martín-Rodríguez S, Loturco I, Hunter AM, Rodríguez-Ruiz D, Munguia-Izquierdo D. 2017. Reliability and Measurement Error of Tensiomyography to Assess Mechanical Muscle Function: A Systematic Review. Journal of Strength and Conditioning Research 31:3524-3536. DOI: 10.1519/JSC.0000000000002250. 
493 Martins J, da Silva JR, da Silva MRB, Bevilaqua-Grossi D. 2017. Reliability and Validity of the 494 Belt-Stabilized Handheld Dynamometer in Hip- and Knee-Strength Tests. Journal of Athletic Training 52:809-819. DOI: 10.4085/1062-6050-52.6.04.

496

497

498

499

500

501

502

503

504

505

506

507

508

509

510

511

512

513

\section{4}

515

516

517

518

Martín-San Agustín R, Benítez-Martínez JC, Medina-Mirapeix F, Casaña-Granell J. 2018. Sex Differences and Patterns of Muscle Stiffness in the Knee Flexor and Extensor Musculature Through Analysis of Isolated Bellies. Journal of Strength and Conditioning Research. DOI: 10.1519/JSC.0000000000002883.

Melchiorri G, Rainoldi A. 2011. Muscle fatigue induced by two different resistances: Elastic tubing versus weight machines. Journal of Electromyography and Kinesiology: Official Journal of the International Society of Electrophysiological Kinesiology 21:954-959. DOI: 10.1016/j.jelekin.2011.07.015.

Menaspà P, Sassi A, Impellizzeri FM. 2010. Aerobic fitness variables do not predict the professional career of young cyclists. Medicine and Science in Sports and Exercise 42:805-812. DOI: 10.1249/MSS.0b013e3181ba99bc.

Montgomery WH, Pink M, Perry J. 1994. Electromyographic analysis of hip and knee musculature during running. The American Journal of Sports Medicine 22:272-278. DOI: 10.1177/036354659402200220.

Navarro-Pujalte E, Gacto-Sánchez M, Montilla-Herrador J, Escolar-Reina P, Ángeles FrancoSierra M, Medina-Mirapeix F. 2018. Sensitivity to change of mobility measures in musculoskeletal conditions on lower extremities in outpatient rehabilitation settings. Disability and Rehabilitation:1-7. DOI: 10.1080/09638288.2018.1424948.

Pardasaney PK, Latham NK, Jette AM, Wagenaar RC, Ni P, Slavin MD, Bean JF. 2012. Sensitivity to change and responsiveness of four balance measures for communitydwelling older adults. Physical Therapy 92:388-397. DOI: 10.2522/ptj.20100398.

de Paula Simola RÁ, Harms N, Raeder C, Kellmann M, Meyer T, Pfeiffer M, Ferrauti A. 2015. Assessment of neuromuscular function after different strength training protocols using 
519

520

521

522

523

524

525

526

527

528

529

530

531

532

533

534

535

536

537

538

539

540

541 Thorlund JB, Michalsik LB, Madsen K, Aagaard P. 2008. Acute fatigue-induced changes in

542 muscle mechanical properties and neuromuscular activity in elite handball players 543 following a handball match. Scandinavian Journal of Medicine \& Science in Sports 544 18:462-472. DOI: 10.1111/j.1600-0838.2007.00710.x.

tensiomyography. Journal of Strength and Conditioning Research 29:1339-1348. DOI: 10.1519/JSC.0000000000000768.

de Paula Simola RÁ, Raeder C, Wiewelhove T, Kellmann M, Meyer T, Pfeiffer M, Ferrauti A. 2016. Muscle mechanical properties of strength and endurance athletes and changes after one week of intensive training. Journal of Electromyography and Kinesiology: Official Journal of the International Society of Electrophysiological Kinesiology 30:73-80. DOI: $10.1016 /$ j.jelekin.2016.05.005.

Raasch CC, Zajac FE, Ma B, Levine WS. 1997. Muscle coordination of maximum-speed pedaling. Journal of Biomechanics 30:595-602.

Raeder C, Wiewelhove T, Simola RÁDP, Kellmann M, Meyer T, Pfeiffer M, Ferrauti A. 2016. Assessment of Fatigue and Recovery in Male and Female Athletes After 6 Days of Intensified Strength Training. Journal of Strength and Conditioning Research 30:34123427. DOI: 10.1519/JSC.0000000000001427.

Rey E, Lago-Peñas C, Lago-Ballesteros J. 2012. Tensiomyography of selected lower-limb muscles in professional soccer players. Journal of Electromyography and Kinesiology: Official Journal of the International Society of Electrophysiological Kinesiology 22:866872. DOI: 10.1016/j.jelekin.2012.06.003.

Tabachnick BG, Fidell LS. 2007. Using multivariate statistics. Boston: Pearson/Allyn \& Bacon. Tam N, Coetzee DR, Ahmed S, Lamberts RP, Albertus-Kajee Y, Tucker R. 2017. Acute fatigue negatively affects risk factors for injury in trained but not well-trained habitually shod runners when running barefoot. European Journal of Sport Science 17:1220-1229. DOI: 10.1080/17461391.2017.1358767. 
545 Tous-Fajardo J, Moras G, Rodríguez-Jiménez S, Usach R, Doutres DM, Maffiuletti NA. 2010.

546 Inter-rater reliability of muscle contractile property measurements using non-invasive

547 tensiomyography. Journal of Electromyography and Kinesiology: Official Journal of the

548 International Society of Electrophysiological Kinesiology 20:761-766. DOI:

$549 \quad$ 10.1016/j.jelekin.2010.02.008.

550 Valencic V, Knez N. 1997. Measuring of skeletal muscles' dynamic properties. Artificial Organs $551 \quad 21: 240-242$.

552 Wiewelhove T, Raeder C, de Paula Simola RA, Schneider C, Döweling A, Ferrauti A. 2017.

553 Tensiomyographic Markers Are Not Sensitive for Monitoring Muscle Fatigue in Elite

$554 \quad$ Youth Athletes: A Pilot Study. Frontiers in Physiology 8:406. DOI:

$555 \quad$ 10.3389/fphys.2017.00406.

556 Wiewelhove T, Schneider C, Döweling A, Hanakam F, Rasche C, Meyer T, Kellmann M, Pfeiffer

557 M, Ferrauti A. 2018. Effects of different recovery strategies following a half-marathon on

558 fatigue markers in recreational runners. PLOS ONE 13:e0207313. DOI:

$559 \quad$ 10.1371/journal.pone.0207313.

560 Zebis MK, Bencke J, Andersen LL, Alkjaer T, Suetta C, Mortensen P, Kjaer M, Aagaard P.

561 2011. Acute fatigue impairs neuromuscular activity of anterior cruciate ligament-agonist

562 muscles in female team handball players. Scandinavian Journal of Medicine \& Science

563 in Sports 21:833-840. DOI: 10.1111/j.1600-0838.2010.01052.x.

564 


\section{Table $\mathbf{1}$ (on next page)}

Baseline characteristics of the participants in total and separated by fatigued condition.

Date represents mean and standard deviation unless otherwise noted. BMI: body mass index; Dm: maximal radial displacement; Tc, contraction time; Vc: contraction velocity between $10-90 \%$ of the Dm; V10: contraction velocity of the first 10\% of the Dm; QF: quadriceps femoris; MVIC: maximal voluntary isometric contraction 


\begin{tabular}{|c|c|c|c|}
\hline $\begin{array}{c}\text { Baseline } \\
\text { Characteristics }\end{array}$ & Total $(\mathrm{n}=39)$ & $\begin{array}{c}\text { Fatigued } \\
\text { participants }(\mathrm{n}=35)\end{array}$ & $\begin{array}{c}\text { Non-fatigued } \\
\text { participants }(n=4)\end{array}$ \\
\hline $\begin{array}{c}\text { Males/females, } \mathrm{N} \\
(\%)\end{array}$ & $19(48.7 \%) / 20(51.3 \%)$ & $\begin{array}{c}16(45.7 \%) / 19 \\
(54.3 \%)\end{array}$ & $3(75 \%) / 1(25 \%)$ \\
\hline Age (years) & $22(2)$ & $22(2)$ & $21(1)$ \\
\hline $\begin{array}{l}\text { Physical activity } \\
\text { (minutes) }\end{array}$ & $316.5(180.8)$ & $314.6(186.7)$ & $332.5(136.9)$ \\
\hline \multicolumn{4}{|l|}{ Anthropometric } \\
\hline Body mass (kg) & $67.37(13.42)$ & $66.10(11.12)$ & 78.55 (12.05) \\
\hline Stature $(\mathrm{cm})$ & $173.3(9.50)$ & $172.5(9.09)$ & $180.7(11.24)$ \\
\hline BMI (kg/m2) & $22.22(2.72)$ & $22.02(2.71)$ & $24(2.53)$ \\
\hline \multicolumn{4}{|l|}{ QF strength } \\
\hline $\operatorname{MVIC}\left(\mathrm{N}^{*} \mathrm{~m}\right)$ & $207.56(74.19)$ & $203.31(75.82)$ & $244.72(50.24)$ \\
\hline \multicolumn{4}{|l|}{$\begin{array}{c}\text { Tensiomyography } \\
\text { parameters }\end{array}$} \\
\hline \multicolumn{4}{|l|}{ Rectus femoris } \\
\hline $\mathrm{Dm}(\mathrm{mm})$ & $10.26(1.42)$ & $10.32(1.44)$ & $9.76(1.28)$ \\
\hline $\mathrm{Tc}(\mathrm{ms})$ & $25.45(4.04)$ & $25.69(3.95)$ & $23.39(4.84)$ \\
\hline $\mathrm{V}_{\mathrm{c}}(\mathrm{mm} / \mathrm{s})$ & $327.96(58.59)$ & $326.62(69.76)$ & $339.70(53.04)$ \\
\hline $\mathrm{V} 10(\mathrm{~mm} / \mathrm{s})$ & $43.07(5.32)$ & $43.08(5.39)$ & $42.93(5.33)$ \\
\hline \multicolumn{4}{|l|}{ Vastus lateralis } \\
\hline $\mathrm{Dm}(\mathrm{mm})$ & $5.74(1.11)$ & $5.63(0.94)$ & $6.64(2.04)$ \\
\hline $\mathrm{Tc}(\mathrm{ms})$ & $21.37(3.02)$ & $21.54(3.11)$ & $19.87(1.35)$ \\
\hline $\mathrm{V}_{\mathrm{c}}(\mathrm{mm} / \mathrm{s})$ & $217.78(50.10)$ & $211.58(39.81)$ & $271.95(97.28)$ \\
\hline $\mathrm{V} 10(\mathrm{~mm} / \mathrm{s})$ & $25.31(5.18)$ & $24.73(4.21)$ & $30.46(9.98)$ \\
\hline \multicolumn{4}{|l|}{ Vastus medialis } \\
\hline $\mathrm{Dm}(\mathrm{mm})$ & $4.57(0.85)$ & $4.52(0.64)$ & $5.08(2.01)$ \\
\hline Tc (ms) & $19.60(1.82)$ & $19.61(1.90)$ & $19.48(1.04)$ \\
\hline $\mathrm{V}_{\mathrm{c}}(\mathrm{mm} / \mathrm{s})$ & $187.22(33.12)$ & $185.08(26.57)$ & $205.93(73.31)$ \\
\hline $\mathrm{V} 10(\mathrm{~mm} / \mathrm{s})$ & $23.22(4.03)$ & $22.97(2.89)$ & $25.37(10.19)$ \\
\hline
\end{tabular}




\section{Table 2 (on next page)}

Differences within and between sex groups in the TMG parameters and MVIC after fatigue protocol.

SD: standard deviation; Dm: maximal radial displacement; Tc, contraction time; Vc:

contraction velocity between $10-90 \%$ of the Dm; V10: contraction velocity of the first $10 \%$ of the Dm; QF: quadriceps femoris; MVIC: maximal voluntary isometric contraction. 


\begin{tabular}{|c|c|c|c|c|c|c|c|c|}
\hline \multirow{3}{*}{ Muscle } & \multicolumn{4}{|c|}{ Males } & \multicolumn{4}{|c|}{ Females } \\
\hline & \multirow{2}{*}{ Baseline } & \multirow{2}{*}{ Fatigued } & \multicolumn{2}{|l|}{ Differences } & \multirow{2}{*}{ Baseline } & \multirow{2}{*}{ Fatigued } & \multicolumn{2}{|l|}{ Differences } \\
\hline & & & Mean (SD); $p$ & $\%$ & & & Mean (SD); $p$ & $\%$ \\
\hline \multicolumn{9}{|l|}{ QF strength } \\
\hline $\operatorname{MVIC}\left(\mathrm{N}^{*} \mathrm{~m}\right)$ & $272.1(51.0)$ & $187.3(40.1)$ & $84.7(37.8) ;<0.001$ & 30.8 & $145.4(30.7)$ & $98.1(24.4)$ & $47.3(22.3) ;<0.001$ & 32.1 \\
\hline \multicolumn{9}{|l|}{ Rectus femoris } \\
\hline $\mathrm{Dm}(\mathrm{mm})$ & $9.91(1.66)$ & $7.46(1.87)$ & $2.45(1.27) ;<0.001$ & 25.2 & $10.67(1.16)$ & $8.71(1.76)$ & $1.95(1.13) ;<0.001$ & 18.7 \\
\hline $\mathrm{Tc}(\mathrm{ms})$ & $24.58(4.25)$ & $24.52(6.37)$ & 0.06 (3.28); 0.941 & 1.1 & $26.62(3.52)$ & $27.63(5.43)$ & $-1.01(4.42) ; 0.334$ & 4.1 \\
\hline $\mathrm{Vc}(\mathrm{mm} / \mathrm{s})$ & 330.01 (78.95) & $250.71(66.81)$ & $79.30(48.65) ;<0.001$ & 21.8 & $373.76(39.15)$ & $256.21(51.02)$ & $67.55(42.26) ;<0.001$ & 20.9 \\
\hline $\mathrm{V} 10(\mathrm{~mm} / \mathrm{s})$ & $43.17(6.55)$ & $32.78(7.72)$ & $10.39(5.35) ;<0.001$ & 24.4 & $43.01(4.37)$ & $33.01(5.13)$ & $10.00(4.20) ;<0.001$ & 23.2 \\
\hline \multicolumn{9}{|l|}{ Vastus lateralis } \\
\hline $\mathrm{Dm}(\mathrm{mm})$ & $5.47(1.18)$ & $4.48(0.76)$ & 0.99 (1.10); 0.003 & 20.5 & $5.78(0.70)$ & $4.10(1.15)$ & $1.68(0.90) ;<0.001$ & 29.5 \\
\hline $\mathrm{Tc}(\mathrm{ms})$ & $21.69(3.05)$ & $19.93(4.31)$ & $1.76(2.44) ; 0.011$ & 8.6 & $21.42(3.24)$ & $19.04(1.88)$ & $2.38(2.15) ;<0.001$ & 10.4 \\
\hline $\mathrm{Vc}(\mathrm{mm} / \mathrm{s})$ & $203.67(49.77)$ & $179.33(66.24)$ & 24.35 (43.77); 0.042 & 12.8 & $218.24(28.76)$ & $170.24(37.41)$ & $48.00(43.15) ;<0.001$ & 20.9 \\
\hline $\mathrm{V} 10(\mathrm{~mm} / \mathrm{s})$ & $24.28(5.04)$ & $20.46(6.78)$ & 3.82 (4.33); 0.003 & 17.3 & $25.10(3.45)$ & $18.65(4.66)$ & $6.45(4.55) ;<0.001$ & 25.3 \\
\hline \multicolumn{9}{|l|}{ Vastus medialis } \\
\hline $\mathrm{Dm}(\mathrm{mm})$ & $4.69(3.91)$ & $3.91(0.78)$ & $0.78(0.59) ;<0.001$ & 16.3 & $4.37(0.50)$ & $3.51(0.69)$ & $0.86(0.53) ;<0.001$ & 19.8 \\
\hline $\mathrm{Tc}(\mathrm{ms})$ & $20.25(1.78)$ & $19.96(2.66)$ & 0.28 (1.97); 0.573 & 1.4 & $19.07(1.88)$ & $18.26(1.88)$ & $0.81(1.64) ; 0.045$ & 3.9 \\
\hline $\mathrm{Vc}(\mathrm{mm} / \mathrm{s})$ & 186.06 (30.93) & $159.90(25.72)$ & $29.16(22.46) ;<0.001$ & 14.9 & $184.26(23.12)$ & $153.76(29.26)$ & $30.50(26.86) ;<0.001$ & 16.2 \\
\hline $\mathrm{V} 10(\mathrm{~mm} / \mathrm{s})$ & $23.76(3.19)$ & 21.09 (3.95) & 2.67 (2.97); 0.003 & 11.2 & $22.31(2.51)$ & $18.33(3.40)$ & $3.98(2.74) ;<0.001$ & 17.8 \\
\hline
\end{tabular}




\section{Table 3 (on next page)}

Responsiveness statistics for the TMG parameters

SRM: standardized response mean; Cl: confidence interval; MCD: minimal detectable change; SE: standard error; AUC: area under curve; Dm: maximal radial displacement; TC, contraction time; Vc: contraction velocity between $10-90 \%$ of the Dm; V10: contraction velocity of the first $10 \%$ of the Dm. + Adjusted by sex 


\begin{tabular}{|c|c|c|c|c|c|c|c|}
\hline \multirow{3}{*}{ Muscle } & \multicolumn{3}{|c|}{ Internal responsiveness } & \multicolumn{4}{|c|}{ External responsiveness } \\
\hline & \multirow{2}{*}{$\begin{array}{c}\text { Paired } \\
\text { t-test } \\
(p) \\
\end{array}$} & \multirow{2}{*}{$\begin{array}{c}\text { SRM } \\
(95 \% \text { CI })\end{array}$} & \multirow{2}{*}{$\begin{array}{c}\% \\
\mathrm{MCD}\end{array}$} & \multirow{2}{*}{$\begin{array}{c}\text { Correlation method } \\
\text { (Pearson's } \mathrm{r} \text { and } 95 \% \\
\mathrm{CI}) ; p \\
\end{array}$} & \multicolumn{2}{|c|}{ Linear regression method +} & \multirow[t]{2}{*}{$\operatorname{AUC}(95 \% \mathrm{CI})$} \\
\hline & & & & & $\mathrm{b}(\mathrm{SE}) ; p$ & $\mathrm{r} 2$ & \\
\hline \multicolumn{8}{|c|}{ Rectus femoris } \\
\hline $\mathrm{Dm}(\mathrm{mm})$ & 0.001 & $-1.83(-2.31 ;-1.47)$ & 91.3 & $0.42(0.12 ; 0.65) ; 0.004$ & $0.40(0.14) ; 0.007$ & 0.22 & $0.73(0.57 ; 0.86)$ \\
\hline Tc (ms) & 0.439 & $0.13(-0.24 ; 0.39)$ & 15.9 & $0.10(-0.22 ; 0.40) ; 0.276$ & $0.14(0.15) ; 0.363$ & 0.06 & $0.62(0.45 ; 0.77)$ \\
\hline $\mathrm{V}_{\mathrm{c}}(\mathrm{mm} / \mathrm{s})$ & 0.001 & $-1.65(-1.98 ;-1.30)$ & 79.7 & $0.33(0.02 ; 0.58) ; 0.020$ & $0.26(0.13) ; 0.052$ & 0.13 & $0.59(0.42 ; 0.74)$ \\
\hline $\mathrm{V} 10(\mathrm{~mm} / \mathrm{s})$ & 0.001 & $-2.20(-2.65 ;-1.78)$ & 97.1 & $0.45(0.15 ; 0.67) ; 0.002$ & $0.43(0.15) ; 0.006$ & 0.22 & $0.73(0.57 ; 0.86)$ \\
\hline \multicolumn{8}{|c|}{ Vastus lateralis } \\
\hline $\mathrm{Dm}(\mathrm{mm})$ & 0.001 & $-1.33(-1.74 ;-0.82)$ & 79.7 & $0.18(-0.14 ; 0.47) ; 0.133$ & $0.10(0.12) ; 0.403$ & 0.05 & $0.81(0.65 ; 0.92)$ \\
\hline Tc (ms) & 0.001 & $-0.87(-1.27 ;-0.41)$ & 65.2 & $0.12(-0.12 ; 0.48) ; 0.111$ & $0.23(0.19) ; 0.238$ & 0.07 & $0.92(0.79 ; 0.98)$ \\
\hline $\mathrm{Vc}(\mathrm{mm} / \mathrm{s})$ & 0.001 & $-0.86(-1.21 ;-0.46)$ & 43.5 & $0.09(-0.23 ; 0.39) ; 0.298$ & $0.03(0.11) ; 0.782$ & 0.04 & $0.55(0.39 ; 0.71)$ \\
\hline $\mathrm{V} 10(\mathrm{~mm} / \mathrm{s})$ & 0.001 & $-1.17(-1.56 ;-0.71)$ & 68.1 & $0.12(-0.20 ; 0.42) ; 0.224$ & $0.06(0.12) ; 0.638$ & 0.04 & $0.67(0.50 ; 0.81)$ \\
\hline \multicolumn{8}{|l|}{$\begin{array}{l}\text { Vastus } \\
\text { medialis }\end{array}$} \\
\hline $\mathrm{Dm}(\mathrm{mm})$ & 0.001 & $-1.46(-1.84 ;-1.07)$ & 76.8 & $0.12(-0.21 ; 0.42) ; 0.116$ & $0.09(0.20) ; 0.643$ & 0.04 & $0.65(0.48 ; 0.79)$ \\
\hline $\mathrm{Tc}(\mathrm{ms})$ & 0.069 & $-0.34(-0.72 ; 0.02)$ & 42 & $-0.14(-0.43 ; 0.18) ; 0.200$ & $-0.28(0.28) ; 0.331$ & 0.06 & $0.52(0.36 ; 0.68)$ \\
\hline $\mathrm{Vc}(\mathrm{mm} / \mathrm{s})$ & 0.001 & $-1.17(-1.50 ;-0.79)$ & 68.1 & $0.17(-0.15 ; 0.46) ; 0.143$ & $0.17(0.19) ; 0.364$ & 0.06 & $0.68(0.52 ; 0.82)$ \\
\hline $\mathrm{V} 10(\mathrm{~mm} / \mathrm{s})$ & 0.001 & $-1.14(-1.47 ;-0.76)$ & 71 & $0.26(-0.06 ; 0.53) ; 0.054$ & $0.25(0.19) ; 0.194$ & 0.08 & $0.76(0.60 ; 0.88)$ \\
\hline
\end{tabular}




\section{Figure 1}

Schematic representation of experimental procedures.

TMG: tensiomyography; MVIC: maximal voluntary isometric contraction.

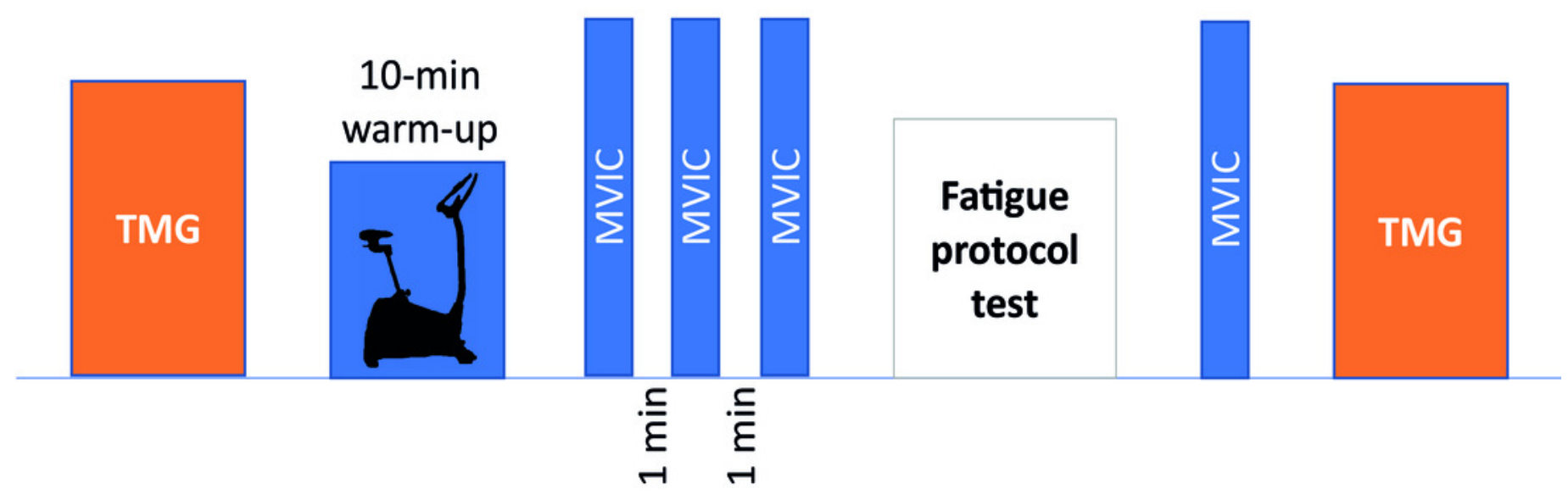




\section{Figure 2}

Tensiomyographical measurement of rectus femoris.

Photo credit: Rodrigo Martín-San Agustín

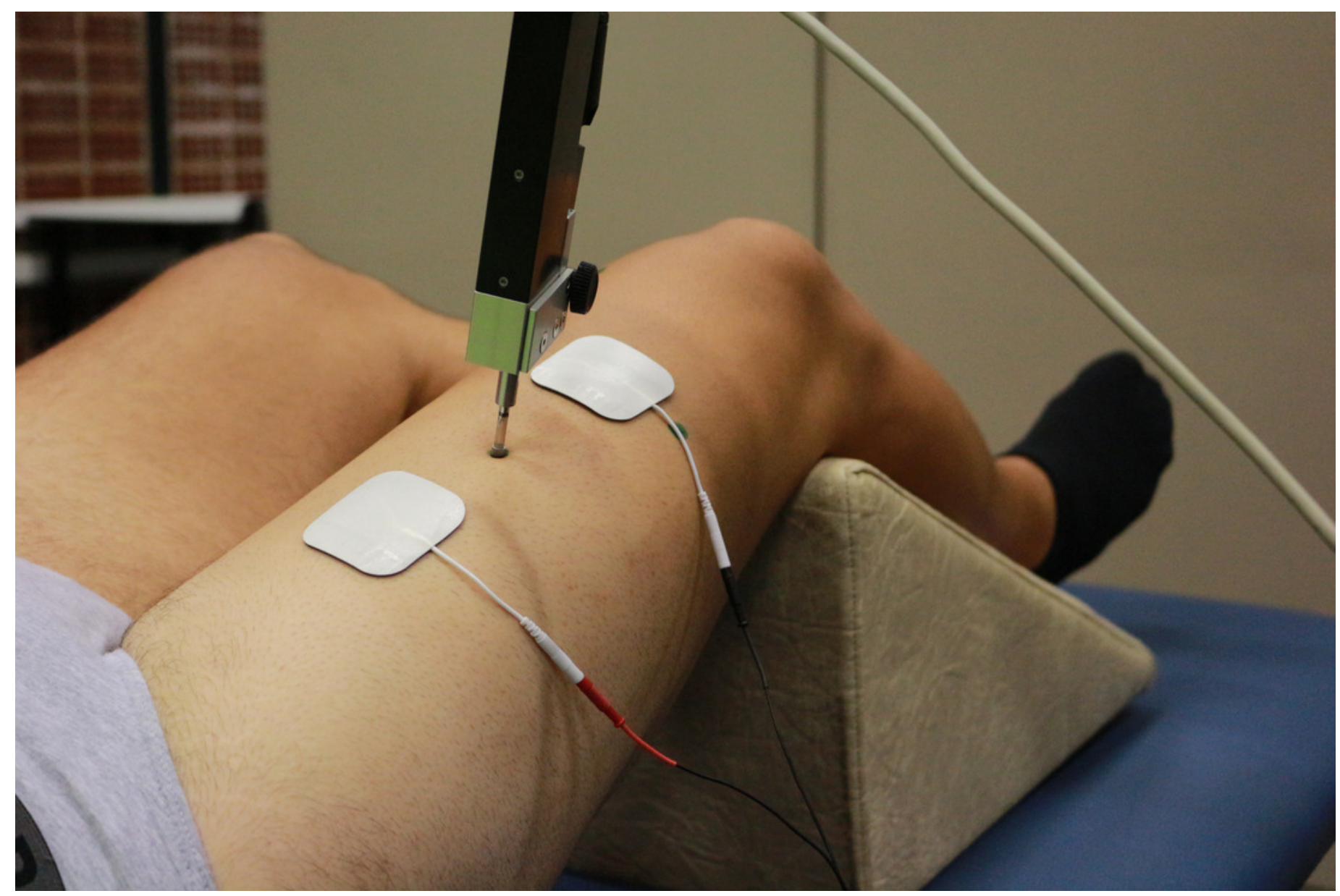


Figure 3

Maximal voluntary isometric contraction test for quadriceps femoris.

Photo credit: Rodrigo Martín-San Agustín 


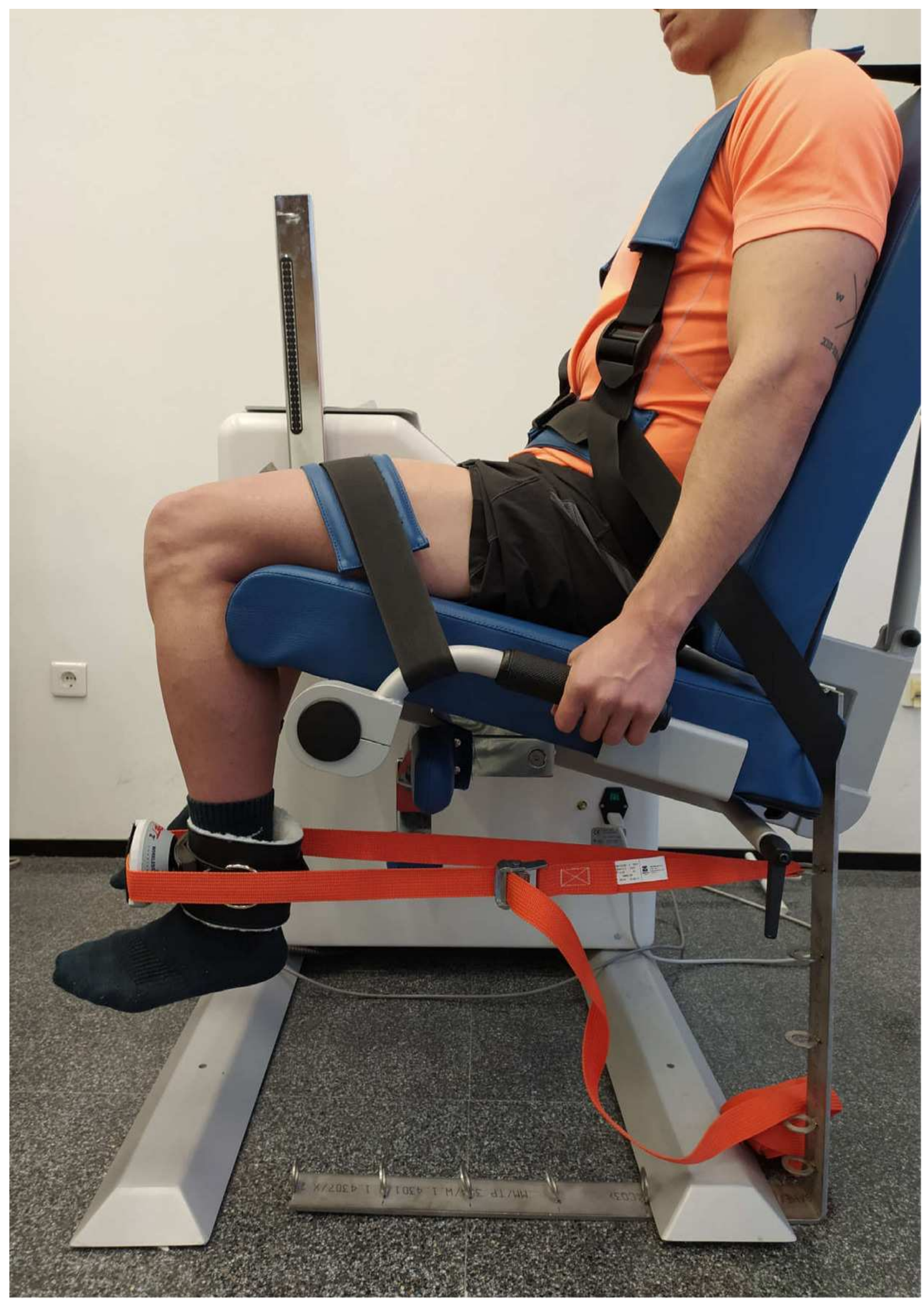

Peer) reviewing PDF | (2019:12:44194:1:1:NEW 23 Jan 2020) 
Figure 4

Differences in TMG parameters of quadriceps bellies between pre- and post-fatigue in all participants.

(A) Differences in Dm, (B) in TC, (C) in VC, and (D) in V10. *Significant differences set at $p<0.01$; Specific $p$-values are shown in table 3 . 

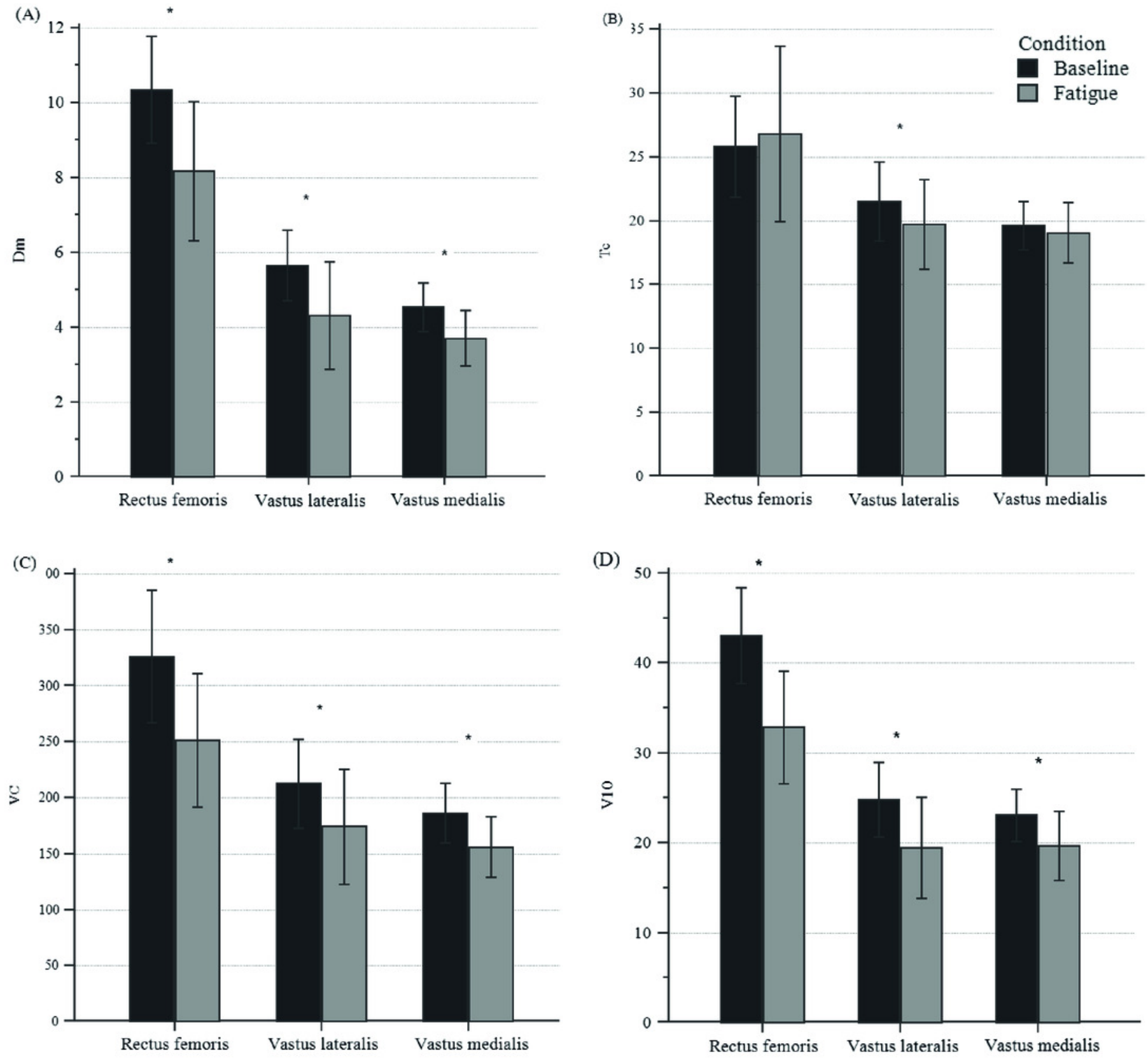